\title{
Optimal control of network-coupled subsystems: Spectral decomposition and low-dimensional solutions
}

\author{
Shuang Gao Member, IEEE, and Aditya Mahajan Senior Member, IEEE
}

\begin{abstract}
In this paper, we investigate optimal control of network-coupled subsystems where the dynamics and the cost couplings depend on an underlying undirected weighted graph. The graph coupling matrix in the dynamics may be the adjacency matrix, the Laplacian matrix, or any other symmetric matrix corresponding to the underlying graph. The cost couplings can be any polynomial function of the underlying coupling matrix We use the spectral decomposition of the graph coupling matrix to decompose the overall system into $(L+1)$ systems with decoupled dynamics and cost, where $L$ is the rank of the coupling matrix. Furthermore, the optimal control input at each subsystem can be computed by solving $\left(L_{\text {dist }}+1\right)$ decoupled Riccati equations where $L_{\text {dist }}\left(L_{\text {dist }} \leq L\right)$ is the number of distinct non-zero eigenvalues of the coupling matrix. A salient feature of the result is that the solution complexity depends on the number of distinct eigenvalues of the coupling matrix rather than the size of the network. Therefore, the proposed solution framework provides a scalable method for synthesizing and implementing optimal control laws for large-scale network-coupled subsystems.
\end{abstract}

\section{INTRODUCTION}

\section{A. Motivation}

The recent proliferation of low cost sensors and actuators has given rise to many networked control systems such as the Internet of Things, smart grids, smart buildings, etc., where multiple subsystems are connected over a network. In such systems, the evolution of the state of a subsystem depends on its local state and local control input and is also influenced by the states and controls of its neighbors. Such networks are often referred to as large-scale systems or complex networks, and various aspects of such systems have been investigated since the early 1970s [1], [2], including issues such as controllability [3], [4], observability [4], [5], control energy metric [6], distributed control [7]-[11], decentralized control [12]-[15] and adaptive control [15].

A key theme for investigating large-scale networked control systems is to identify conditions under which the optimal control laws may be synthesized and implemented with lowcomplexity. Such conditions include simplified control objectives (e.g., consensus [7]-[11] and synchronization [16]), sim-

S. Gao and A. Mahajan are with the Department of Electrical and Computer Engineering, McGill University, Montreal, QC, Canada. Emails sgao@cim.mcgill.ca, aditya.mahajan@mcgill.ca.

The work of the second author was supported in part by the Innovation for Defence Excellence and Security (IDEaS) Program of the Canadian Department of National Defence through grant CFPMN2-30

Preliminary version of this work was presented at the 58th IEEE Conference on Decision and Control, Nice, France, December, 2019. plified control inputs (e.g., pinning control [17]-[19] and ensemble control [20]), simplified coupling between subsystems (e.g., symmetric interconnections [4], [12], [13], [21], [22], exchangeable or anonymous subsystems [23]-[26], sparse connections or structure reduction [27], [28], hierarchical decompositions [29] and patterned systems [30]), approximate optimality (e.g., mean-field games [31]-[34], control based on approximate aggregations [35], approximate distributed control [36], [37], and graphon control [38], [39]).

In this paper, we propose a decomposition method for largescale network-coupled subsystems which relies on the spectral decomposition of the dynamic and cost coupling between the subsystems. Several related approaches have been considered in the literature. An earlier approach similar in spirit to ours is [35], which considered the problem of approximating a high-dimensional system with a low-dimensional system using what was called state aggregation. Both exact and approximate solutions were proposed. Spectral decomposition of large-scale systems with symmetric interconnected subsystems have been considered in [12], [13]. Algebraic decomposition of meanfield coupled subsystems has been considered in [24]-[26]. A key feature which distinguishes our approach from these previous work is that our approach is applicable to models where the coupling between agents are not homogeneous and that we establish optimal solutions rather than an approximate solution. Another line of related work is graphical games [27], [40, Chapter 6] where the coupling of the utility function depends on an underlying graph. In contrast to these, we consider a control problem and propose a different type of decomposition.

\section{B. Contributions of this paper}

In this paper, we investigate a control system with multiple subsystems connected over an undirected graph. Each subsystem has a local state and takes a local control action. The evolution of the state of each subsystem depends on its local state and local control as well as a weighted combination (which we call the network field) of the states and controls of its neighbors. Moreover, the weights in the network field, which are represented by a coupling matrix, may correspond to the adjacency matrix, Laplacian matrix or any other symmetric matrix that characterizes the underlying graph. Each subsystem is also coupled to its multi-hop neighbors via a quadratic cost. The cost couplings can be any polynomial function of the coupling matrix of the underlying graph. The objective is to 
choose the control inputs of each subsystem to minimize the total cost over time. The above model is a linear quadratic regulation problem and a centralized solution can be obtained by solving $n d_{x} \times n d_{x}$-dimensional Riccati equation, where $n$ is the number of subsystems and $d_{x}$ is the dimension of the state of each subsystem. In this paper, we propose an alternative solution that has low complexity and may be implemented in a local manner with aggregated (or projected) state information and local information. For some particular cases, the control can be implemented in a distributed manner that relies on neighbourhood information and local information.

The main contributions of our paper are the following:

- A spectral decomposition technique is devoloped to decomposes the linear quadratic control problem for network-coupled dynamical subsystems into $L+1$ decoupled subproblems, where $L$ is the rank of the coupling matrix.

- These $L+1$ decoupled subproblems can be solved by solving only $L_{\text {dist }}+1$ decoupled Riccati equations of dimension $d_{x} \times d_{x}$, where $L_{\text {dist }}$ is the number of distinct non-zero eigenvalues of the coupling matrix and $d_{x}$ is the state dimension of each subsystem. In contrast, a direct centralized solution requires solving an $n d_{x} \times n d_{x^{-}}$ dimensional Riccati equation where $n$ is the number of subsystems. We note that for any coupling matrix, the inequalities $L_{\text {dist }} \leq L \leq n$ always hold. Thus the method proposed in this paper leads to considerable simplifications in synthesizing optimal control laws.

- To implement the optimal control input, each subsystem needs to know the $(L+1) d_{x}$-dimensional vector of local components of eigen and auxiliary states (which are defined later in the paper). In contrast, to implement the centralized solution, each subsystem needs to know the $n d_{x}$ dimensional global state. Thus, in applications such as [41]-[44] where $L \ll n$, the method proposed in this paper leads to considerable simplification in implementing the optimal control law.

- The solution method is extended to solve stochastic linear quadratic control problems for network-coupled subsystems.

- The solution method is applied to study consensus problems to establish optimal distributed control solutions for some particular cases.

\section{Notations and definitions}

We use $\mathbb{N}$ and $\mathbb{R}$ to denote respectively the sets of natural and real numbers. The notation $A=\left[a_{i j}\right]$ means that $a_{i j}$ is the $(i, j)$ th element of the matrix $A$. For a vector $v, v_{i}$ denotes its $i$ th element. For a matrix $A, A^{\top}$ denotes its transpose. Given vectors $v^{1}, \ldots, v^{n}, \operatorname{cols}\left(v^{1}, \ldots, v^{n}\right)$ denotes the matrix formed by horizontally stacking the vectors. For any $n \in \mathbb{N}$, $\mathbb{1}_{n}$ denotes the $n$-dimensional vector of ones, $\mathbb{1}_{n \times n}$ denotes the $n \times n$-dimensional matrix of ones, and $I_{n}$ denotes the $n \times n$-dimensional identity matrix.

A pair $(A, B)$ is stabilizable if there exists a matrix $L$ such that $A+B L$ is Hurwitz (i.e., all its eigenvalues have negative real parts). A pair $(A, C)$ is detectable if there exists a matrix $F$ such that $A+F C$ is Hurwitz.

\section{SYSTEM MODEL AND PROBLEM FORMULATION}

\section{A. System model}

Consider a network consisting of $n$ nodes connected over an undirected weighted graph $\mathcal{G}(\mathcal{N}, \mathcal{E}, W)$, where $\mathcal{N}=$ $\{1, \ldots, n\}$ is the set of nodes, $\mathcal{E} \subseteq \mathcal{N} \times \mathcal{N}$ is the unordered set of edges, and $W=\left[w_{i j}\right] \in \mathbb{R}^{n \times n}$ is the weighted adjacency matrix. Let $M=\left[m_{i j}\right] \in \mathbb{R}^{n \times n}$ be some general symmetric coupling matrix corresponding to the underlying graph $\mathcal{G}(\mathcal{N}, \mathcal{E}, W)$. For instance, $M$ may represent the underlying adjacency matrix (i.e., $M=W$ ) or represent the underlying Laplacian matrix (i.e., $M=\operatorname{diag}\left(W \mathbb{1}_{n}\right)-W$ ). For any node $i \in \mathcal{N}, \mathcal{N}_{i}:=\{j \in \mathcal{N}:(i, j) \in \mathcal{E}\}$ denotes the set of neighbors of node $i$. Note that the edge set $\mathcal{E}$ is allowed to include self-loops. Therefore the set $\mathcal{N}_{i}$ may contain node $i$.

The system operates in continuous time for either a finite interval $[0, T]$ or an infinite interval $[0, \infty)$. A state $x_{i}(t) \in$ $\mathbb{R}^{d_{x}}$ and a control input $u_{i}(t) \in \mathbb{R}^{d_{u}}$ are associated with each node $i \in \mathcal{N}$. At time $t=0$, the system starts from an initial state $\left(x_{i}(0)\right)_{i \in \mathcal{N}}$ and for $t>0$, the state of node $i$ evolves according to

$$
\dot{x}_{i}(t)=A x_{i}(t)+B u_{i}(t)+D x_{i}^{\mathcal{G}}(t)+E u_{i}^{\mathcal{G}}(t),
$$

where $A, B, D$ and $E$ are matrices of appropriate dimensions and

$$
x_{i}^{\mathcal{G}}(t)=\sum_{j \in \mathcal{N}_{i}} m_{i j} x_{j}(t) \quad \text { and } \quad u_{i}^{\mathcal{G}}(t)=\sum_{j \in \mathcal{N}_{i}} m_{i j} u_{j}(t)
$$

are the locally perceived network field of states and control actions at node $i$. It is assumed that all the different subsystems have the same parameter matrices $A, B, D$ and $E$.

We follow an atypical representation of the "vectorized" dynamics. Define

$$
\begin{aligned}
& x(t)=\operatorname{cols}\left(x_{1}(t), \ldots, x_{n}(t)\right), \\
& u(t)=\operatorname{cols}\left(u_{1}(t), \ldots, u_{n}(t)\right),
\end{aligned}
$$

as the global state and control actions of the system, and

$$
\begin{aligned}
x^{\mathcal{G}}(t) & =\operatorname{cols}\left(x_{1}^{\mathcal{G}}(t), \ldots, x_{n}^{\mathcal{G}}(t)\right), \\
u^{\mathcal{G}}(t) & =\operatorname{cols}\left(u_{1}^{\mathcal{G}}(t), \ldots, u_{n}^{\mathcal{G}}(t)\right),
\end{aligned}
$$

as the global network field of states and actions. Note that $x(t), x^{\mathcal{G}}(t) \in \mathbb{R}^{d_{x} \times n}$ and $u(t), u^{\mathcal{G}}(t) \in \mathbb{R}^{d_{u} \times n}$ are matrices and not vectors. The system dynamics may be written as

$$
\dot{x}(t)=A x(t)+B u(t)+D x^{\mathcal{G}}(t)+E u^{\mathcal{G}}(t) .
$$

Furthermore, we may write

$$
x^{\mathcal{G}}(t)=x(t) M^{\top}=x(t) M \text { and } u^{\mathcal{G}}(t)=u(t) M^{\top}=u(t) M .
$$

\section{B. System performance and control objective}

At any time $t \in[0, T)$, the system incurs an instantaneous cost

$c(x(t), u(t))=\sum_{i \in \mathcal{N}} \sum_{j \in \mathcal{N}}\left[g_{i j} x_{i}(t)^{\top} Q x_{j}(t)+h_{i j} u_{i}(t)^{\top} R u_{j}(t)\right]$, 
and at the terminal time $T$, the system incurs a terminal cost

$$
c_{T}(x(T))=\sum_{i \in \mathcal{N}} \sum_{j \in \mathcal{N}} g_{i j} x_{i}(T)^{\top} Q_{T} x_{j}(T),
$$

where $Q, Q_{T}$, and $R$ are matrices of appropriate dimensions and $g_{i j}$ and $h_{i j}$ are real-valued weights.

We are interested in the following optimization problems.

Problem 1 Choose a control trajectory $u:[0, T) \rightarrow \mathbb{R}^{d_{u} \times n}$ to minimize

$$
J(u)=\int_{0}^{T} c(x(t), u(t)) d t+c_{T}(x(T))
$$

subject to the dynamics in (3).

Problem 2 Choose a control trajectory $u:[0, \infty) \rightarrow \mathbb{R}^{d_{u} \times n}$ to minimize

$$
J(u)=\int_{0}^{\infty} c(x(t), u(t)) d t .
$$

subject to the dynamics in (3).

\section{Assumptions on the model}

In this section, we describe the assumptions imposed on the model. Let $G=\left[g_{i j}\right]$ and $H=\left[h_{i j}\right]$.

(A0) The weight matrices $G$ and $H$ are polynomials of $M$, i.e., $G=\sum_{k=0}^{K_{G}} q_{k} M^{k}$ and $H=\sum_{k=0}^{K_{H}} r_{k} M^{k}$ where $K_{G}$ and $K_{H}$ denote the degree of the polynomials and $\left\{q_{k}\right\}_{k=0}^{K_{G}}$ and $\left\{r_{k}\right\}_{k=0}^{K_{H}}$ are real-valued coefficients.

Since $M$ is real and symmetric, it has real eigenvalues. Let $L$ denote the rank of $M$ and $\lambda^{1}, \ldots, \lambda^{L}$ denote the non-zero eigenvalues. For ease of notation, for $\ell \in\{1, \ldots, L\}$, define

$$
q^{\ell}=\sum_{k=0}^{K_{G}} q_{k}\left(\lambda^{\ell}\right)^{k} \quad \text { and } \quad r^{\ell}=\sum_{k=0}^{K_{H}} r_{k}\left(\lambda^{\ell}\right)^{k} .
$$

(A1) The matrices $Q$ and $Q_{T}$ are symmetric and positive semidefinite and $R$ is symmetric and positive definite.

(A2) For $\ell \in\{1, \ldots, L\}, q^{\ell}$ is non-negative and $r^{\ell}$ is strictly positive. Moreover $q_{0} \geq 0$ and $r_{0}>0$.

Assumption (A0) ensures that $M, H$ and $G$ share the same eigenvectors, which allows us to decouple the dynamics and the cost based on the same spectral decomposition (see Section III for more details). Assumption (A2) ensures that for any $y \in$ $\mathbb{R}^{n}, y^{\top} G y \geq 0$ and $y^{\top} H y>0$. Assumptions (A1) and (A2) ensure that $G \otimes Q$ and $G \otimes Q_{T}$ are symmetric positive semidefinite, and $H \otimes R$ is symmetric positive definite, which are standard sufficient conditions for finite-horizon LQR problems to have a unique optimal solution (see for instance [45]).

\section{Some remarks on the assumptions on the cost function}

Since the subsystems (or agents) are coupled in the dynamics over an underlying graph, it is reasonable to assume that the cost structure respects the same graph structure. The polynomials allow us to consider cost coupling structures which may involve not only the immediate neighbourhood but also multiple-hop neighbourhood connections.

We present a few examples with different coefficients $\left\{q_{k}\right\}_{k=0}^{K_{G}}$ and $\left\{r_{k}\right\}_{k=0}^{K_{H}}$ below:
1) If $K_{G}=K_{H}=0, q_{0}=1, r_{0}=1$ and all other coefficients are zero, then $G=H=I$. In this case, the instantaneous cost reduces to

$$
c(x(t), u(t))=\sum_{i=1}^{n}\left[x_{i}(t)^{\top} Q x_{i}(t)+u_{i}(t)^{\top} R u_{i}(t)\right] .
$$

Thus, the problem is equivalent to the social optimal control problem where the cost is the summation of the costs of all the subsystems.

2) If $K_{G}=2, K_{H}=1, q_{0}=1, q_{1}=-2, q_{2}=1, r_{0}=1$ and all other coefficients are zero, then $G=(I-M)^{2}$ and $H=1$. If, furthermore, the matrix $M=\frac{1}{n} \mathbb{1}_{n} \mathbb{1}_{n}^{\top}$, then the instantaneous cost reduces to

$$
\begin{gathered}
c(x(t), u(t))=\sum_{i=1}^{n}\left[\left(x_{i}(t)-\bar{x}(t)\right)^{\top} Q\left(x_{i}(t)-\bar{x}(t)\right)\right. \\
\left.+u_{i}(t)^{\top} R u_{i}(t)\right]
\end{gathered}
$$

where $\bar{x}(t) \triangleq \frac{1}{n} \sum_{i=1}^{n} x_{i}(t)$. Thus, the problem is equivalent to the social optimal mean field control problem (similar to the cost considered in [46]).

3) If $K_{G}=2, K_{H}=0, q_{2}=1, r_{0}=1$, all other coefficients are zero, and the coupling matrix is the Laplacian matrix, then

$$
G=M^{2}, H=I, M=\mathcal{L} \triangleq \operatorname{diag}\left(W \mathbb{1}_{n}-W\right) .
$$

Then the instantaneous cost reduces to

$$
(x(t), u(t))=\sum_{i=1}^{n}\left[e_{i}(t)^{\top} Q e_{i}(t)+u_{i}(t)^{\top} R u_{i}(t)\right]
$$

where the local state error for subsystem $i$ is given by

$$
e_{i}(t) \triangleq \sum_{j \in \mathcal{N}_{i}} w_{i j}\left(x_{i}(t)-x_{j}(t)\right)
$$

If, furthermore, the coupling only appears in the cost (i.e., there are no couplings in the dynamics) and $A=0$, this structure then produces the optimal control problem that can be associated with a distributed control problem (see Section $\mathrm{V}$ for more details).

4) $K_{G}$ and $K_{H}$ can be $\infty$ as long as the limit of the corresponding polynomial series is well defined. Such examples include the exponential function $G=$ $\exp (M)=\sum_{k=0}^{\infty} \frac{1}{k !} M^{k}$, and the inverse function $G=$ $(I-\gamma M)^{-1}=\sum_{k=0}^{\infty} \gamma^{k} M^{k}$ (when the spectral radius $\rho(M)$ of $M$ satisfies $\rho(M)<\gamma)$.

\section{E. Salient features of the model}

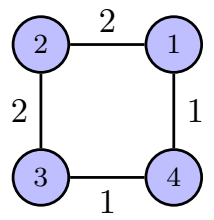

(a) A graph $\mathcal{G}$

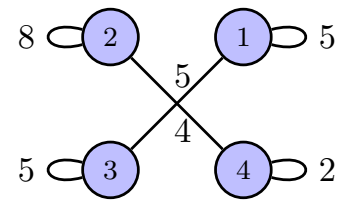

(b) 2-hop neighborhood of $\mathcal{G}$
Fig. 1: A graph and its 2-hop neighborhood. 
We highlight salient features of the model via an example. Consider a system with 4 nodes connected via a network shown in Fig. 11a), with

$$
G=q_{0} I+q_{1} M+q_{2} M^{2} \text { and } H=r_{0} I+r_{1} M+r_{2} M^{2},
$$

where $M$ and $M^{2}$ are the weighted adjacency matrix of the graph $\mathcal{G}$ and that of the 2-hop neighborhood of $\mathcal{G}$, respectively, given by

$$
M=\left[\begin{array}{llll}
0 & 2 & 0 & 1 \\
2 & 0 & 2 & 0 \\
0 & 2 & 0 & 1 \\
1 & 0 & 1 & 0
\end{array}\right] \quad \text { and } \quad M^{2}=\left[\begin{array}{llll}
5 & 0 & 5 & 0 \\
0 & 8 & 0 & 4 \\
5 & 0 & 5 & 0 \\
0 & 4 & 0 & 2
\end{array}\right] .
$$

1) Salient features of the dynamics: For this example,

$$
\begin{array}{ll}
x_{1}^{\mathcal{G}}(t)=2 x_{2}(t)+x_{4}(t), & x_{2}^{\mathcal{G}}(t)=2 x_{1}(t)+2 x_{3}(t), \\
x_{3}^{\mathcal{G}}(t)=2 x_{2}(t)+x_{4}(t), & x_{4}^{\mathcal{G}}(t)=x_{1}(t)+x_{3}(t) .
\end{array}
$$

Thus, each subsystem is affected by its neighbors. The influence of each neighbor is not homogeneous but depends on the weight associated with the corresponding edge in the graph. Furthermore, the network field $x^{\mathcal{G}}(t)$ is not homogeneous and varies from subsystem to subsystem.

2) Salient features of the cost: If $M$ is the weighted adjacency matrix of the graph $\mathcal{G}$, the matrix $M^{k}, k \in \mathbb{N}$, represents the weighted adjacency matrix of the $k$-hop neighborhood of $\mathcal{G}$. Thus, $G=q_{0} I+q_{1} M+q_{2} M^{2}$ means that each node has a coupling of $q_{0}$ with its own state, a coupling of $q_{1}$ with its 1-hop neighborhood and a coupling of $q_{2}$ with its 2-hop neighborhood. Similar interpretation holds for $H$. Note that

$$
G=q_{0} I+q_{1} M+q_{2} M^{2}=\left[\begin{array}{cccc}
q_{0}+5 q_{2} & 2 q_{1} & 5 q_{2} & q_{0}+q_{1} \\
2 q_{1} & q_{0}+8 q_{2} & 2 q_{1} & 4 q_{2} \\
5 q_{2} & 2 q_{1} & q_{0}+5 q_{2} & q_{1} \\
q_{1} & 4 q_{2} & q_{1} & q_{0}+2 q_{2}
\end{array}\right] .
$$

Thus, the agents are not interchangeable, i.e., in general, $G_{i i} \neq$ $G_{j j}$ and $G_{k i} \neq G_{k j}$.

\section{SPECTRAL DECOMPOSITION OF THE SYSTEM}

Since the weight matrix $M$ is real and symmetric, it admits a spectral factorization. In particular, there exist nonzero eigenvalues $\left(\lambda^{1}, \ldots, \lambda^{L}\right)$ and orthonormal eigenvectors $\left(v^{1}, \ldots, v^{L}\right)$ such that

$$
M=\sum_{\ell=1}^{L} \lambda^{\ell} v^{\ell} v^{\ell^{\top}} .
$$

In the rest of this section, we decompose the dynamics and the cost based on the above spectral decomposition. Our decompositions may be viewed as generalizations of mean-field decompositions used in [24]-[26] to heterogenous networks.

\section{A. Spectral decomposition of the dynamics}

For $\ell \in\{1, \ldots, L\}$, define eigenstates and eigencontrol actions as

$$
\begin{aligned}
& x^{\ell}(t)=x(t) v^{\ell} v^{\ell^{\top}}, \\
& u^{\ell}(t)=u(t) v^{\ell} v^{\ell^{\top}},
\end{aligned}
$$

respectively. Multiplying both sides of (3) by $v^{\ell} v^{\ell^{\top}}$, we get

$$
\dot{x}^{\ell}(t)=\left(A+\lambda^{\ell} D\right) x^{\ell}(t)+\left(B+\lambda^{\ell} E\right) u^{\ell}(t),
$$

where we have used the fact that $M v^{\ell} v^{\ell^{\top}}=\lambda^{\ell} v^{\ell} v^{\ell^{\top}}$. Let $x_{i}^{\ell}(t)$ and $u_{i}^{\ell}(t)$ denote the $i$-th column of these matrices, i.e.,

$$
\begin{aligned}
& x^{\ell}(t)=\operatorname{cols}\left(x_{1}^{\ell}(t), \ldots, x_{n}^{\ell}(t)\right), \\
& u^{\ell}(t)=\operatorname{cols}\left(u_{1}^{\ell}(t), \ldots, u_{n}^{\ell}(t)\right) .
\end{aligned}
$$

Therefore, the dynamics (11) can be written as a collection of decoupled "local" dynamics: for $i \in \mathcal{N}$,

$$
\dot{x}_{i}^{\ell}(t)=\left(A+\lambda^{\ell} D\right) x_{i}^{\ell}(t)+\left(B+\lambda^{\ell} E\right) u_{i}^{\ell}(t) .
$$

Using the spectral factorization (8), we may write:

$$
\begin{aligned}
& x^{\mathcal{G}}(t)=x(t) M=\sum_{\ell=1}^{L} \lambda^{\ell} x^{\ell}(t), \\
& u^{\mathcal{G}}(t)=u(t) M=\sum_{\ell=1}^{L} \lambda^{\ell} u^{\ell}(t) .
\end{aligned}
$$

Now, define auxiliary state and control actions as

$$
\breve{x}(t)=x(t)-\sum_{\ell=1}^{L} x^{\ell}(t) \quad \text { and } \quad \breve{u}(t)=u(t)-\sum_{\ell=1}^{L} u^{\ell}(t) .
$$

Then, by subtracting (11) from (3) and substituting (13) and (14), we get

$$
\dot{\breve{x}}(t)=A \breve{x}(t)+B \breve{u}(t) .
$$

Note that $\breve{x}(t) \in \mathbb{R}^{d_{x} \times n}$ and $\breve{u}(t) \in \mathbb{R}^{d_{u} \times n}$. Let $\breve{x}_{i}(t)$ and $\breve{u}_{i}(t)$ denote the $i$-th column of these matrices, i.e.,

$$
\begin{aligned}
\breve{x}(t) & =\operatorname{cols}\left(\breve{x}_{1}(t), \ldots, \breve{x}_{n}(t)\right), \\
\breve{u}(t) & =\operatorname{cols}\left(\breve{u}_{1}(t), \ldots, \breve{u}_{n}(t)\right) .
\end{aligned}
$$

Therefore, the dynamics (15) of the auxiliary state can be written as a collection of decoupled "local" dynamics:

$$
\dot{\vec{x}}_{i}(t)=A \breve{x}_{i}(t)+B \breve{u}_{i}(t), \quad i \in \mathcal{N} .
$$

The above decomposition may be summarized as follows.

Proposition 1 The local state and control at each node $i \in \mathcal{N}$ may be decomposed as

$$
\begin{aligned}
& x_{i}(t)=\breve{x}_{i}(t)+\sum_{\ell=1}^{L} x_{i}^{\ell}(t), \\
& u_{i}(t)=\breve{u}_{i}(t)+\sum_{\ell=1}^{L} u_{i}^{\ell}(t),
\end{aligned}
$$

where the dynamics of $\breve{x}_{i}(t)$ depend on only $\breve{u}_{i}(t)$ and are given by (16) and the dynamics of $x_{i}^{\ell}(t)$ depends on only $u_{i}^{\ell}(t)$ and are given by (12). 


\section{B. Spectral decomposition of the cost}

For any $n \times n$ matrix $P=\left[p_{i j}\right]$, any $d \times n$ matrices $x=\operatorname{cols}\left(x_{1}, \ldots, x_{n}\right)$, and $y=\operatorname{cols}\left(y_{1}, \ldots, y_{n}\right)$, we use the following short hand notation:

$$
\langle x, y\rangle_{P}=\sum_{i \in \mathcal{N}} \sum_{j \in \mathcal{N}} p_{i j} x_{i}^{\top} y_{j}
$$

Proposition 2 The instantaneous cost may be written as

$$
c(x(t), u(t))=\langle x(t), Q x(t)\rangle_{G}+\langle u(t), R u(t)\rangle_{H},
$$

which can be simplified as follows:

$$
\begin{gathered}
\langle x(t), Q x(t)\rangle_{G} \\
=\sum_{i \in \mathcal{N}}\left[q_{0} \breve{x}_{i}(t)^{\top} Q \breve{x}_{i}(t)+\sum_{\ell=1}^{L} q^{\ell} x_{i}^{\ell}(t)^{\top} Q x_{i}^{\ell}(t)\right], \\
\langle u(t), R u(t)\rangle_{H} \\
=\sum_{i \in \mathcal{N}}\left[r_{0} \breve{u}_{i}(t)^{\top} R \breve{u}_{i}(t)+\sum_{\ell=1}^{L} r^{\ell} u_{i}^{\ell}(t)^{\top} R u_{i}^{\ell}(t)\right] .
\end{gathered}
$$

See Appendix for the proof.

\section{The MAIN RESUlts: STRUCTURE AND SYNTHESIS OF OPTIMAL CONTROL STRATEGIES}

\section{A. Finite horizon setup}

The main result for the finite horizon setup is the following.

Theorem 1 For $\ell \in\{1, \ldots, L\}$, let $P^{\ell}:[0, T] \rightarrow \mathbb{R}^{d_{x} \times d_{x}}$ be the solution to the backward Riccati differential equation

$$
\begin{aligned}
& -\dot{P}^{\ell}(t)=\left(A+\lambda^{\ell} D\right)^{\top} P^{\ell}(t)+P^{\ell}(t)\left(A+\lambda^{\ell} D\right) \\
& -P^{\ell}(t)\left(B+\lambda^{\ell} E\right)\left(r^{\ell} R\right)^{-1}\left(B+\lambda^{\ell} E\right)^{\top} P^{\ell}(t)+q^{\ell} Q
\end{aligned}
$$

with the final condition $P^{\ell}(T)=q^{\ell} Q_{T}$. Similarly, let $\breve{P}:[0, T] \rightarrow \mathbb{R}^{d_{x} \times d_{x}}$ be the solution to the backward Riccati differential equation

$$
-\dot{\breve{P}}(t)=A^{\top} \breve{P}(t)+\breve{P}(t) A-\breve{P}(t) B\left(r_{0} R\right)^{-1} B^{\top} \breve{P}(t)+q_{0} Q
$$

with the final condition $\breve{P}(T)=q_{0} Q_{T}$.

Then, under assumptions (A0), (A1) and (A2), the optimal control strategy for Problem 1$]$ is given by

$$
u_{i}(t)=-\breve{K}(t) \breve{x}_{i}(t)-\sum_{\ell=1}^{L} K^{\ell}(t) x_{i}^{\ell}(t), \quad i \in \mathcal{N},
$$

where

$$
\begin{aligned}
\breve{K}(t) & =\left(r_{0} R\right)^{-1} B^{\top} \breve{P}(t), \\
K^{\ell}(t) & =\left(r^{\ell} R\right)^{-1}\left(B+\lambda^{\ell} E\right)^{\top} P^{\ell}(t) .
\end{aligned}
$$

PROOF Consider the following collections of dynamical systems:

- Eigensystem $(\ell, i), \ell \in\{1, \ldots, L\}, i \in \mathcal{N}$, with state $x_{i}^{\ell}(t)$, control inputs $u_{i}^{\ell}(t)$, dynamics

$$
\dot{x}_{i}^{\ell}(t)=\left(A+\lambda^{\ell} D\right) x_{i}^{\ell}(t)+\left(B+\lambda^{\ell} E\right) u_{i}^{\ell}(t),
$$

and cost

$$
\begin{aligned}
J_{i}^{\ell}\left(u_{i}^{\ell}\right)=\int_{0}^{T}\left[q^{\ell} x_{i}^{\ell}(t)^{\top} Q x_{i}^{\ell}(t)\right. & \left.+r^{\ell} u_{i}^{\ell}(t)^{\top} R u_{i}^{\ell}(t)\right] d t \\
& +q^{\ell} x_{i}^{\ell}(T)^{\top} Q x_{i}^{\ell}(T) .
\end{aligned}
$$

- Auxiliary system $i, i \in \mathcal{N}$, with state $\breve{x}_{i}(t)$, control inputs $\breve{u}_{i}(t)$, dynamics

$$
\dot{\breve{x}}_{i}(t)=A \breve{x}_{i}(t)+B \breve{u}_{i}(t),
$$

and cost

$$
\begin{aligned}
\breve{J}_{i}\left(\breve{u}_{i}\right)=\int_{0}^{T}\left[q_{0} \breve{x}_{i}(t)^{\top} Q \breve{x}_{i}(t)\right. & \left.+r_{0} \breve{u}_{i}(t)^{\top} R \breve{u}_{i}(t)\right] d t \\
& +q_{0} \breve{x}_{i}(T)^{\top} Q \breve{x}_{i}(T) .
\end{aligned}
$$

Note that all systems have decoupled dynamics and decoupled nonnegative cost. By Proposition 2] we have

$$
J(u)=\sum_{i \in \mathcal{N}}\left[\breve{J}_{i}\left(\breve{u}_{i}\right)+\sum_{\ell=1}^{L} J_{i}^{\ell}\left(u_{i}^{\ell}\right)\right] .
$$

Thus, instead of solving:

(CP1) choose control trajectory $u:[0, T) \rightarrow \mathbb{R}^{d_{u} \times n}$ to minimize $J(u)$,

we can equivalently solve the following optimal control problems:

(CP2) choose control trajectory $u_{i}^{\ell}:[0, T) \rightarrow \mathbb{R}^{d_{u}}$ to minimize $J_{i}^{\ell}\left(u_{i}^{\ell}\right)$ for $i \in \mathcal{N}, \ell \in\{1, \ldots, L\}$,

(CP3)choose control trajectory $\breve{u}_{i}:[0, T) \rightarrow \mathbb{R}^{d_{u}}$ to minimize $\breve{J}_{i}\left(\breve{u}_{i}\right)$ for $i \in \mathcal{N}$.

Given the solutions of Problems (CP2) and (CP3), we can use Proposition 1 and choose $u_{i}(t)$ according to (18).

Problems (CP2) and (CP3) are standard optimal control problems and their solution are given as follows. Let $P^{\ell}:[0, T] \rightarrow \mathbb{R}^{d_{x} \times d_{x}}$ and $\breve{P}:[0, T] \rightarrow \mathbb{R}^{d_{x} \times d_{x}}$ be as given by (20) and 21). Then, for all $i \in \mathcal{N}$, the optimal solution of (CP2) is given by $u_{i}^{\ell}(t)=K^{\ell}(t) x_{i}^{\ell}(t), \ell \in\{1, \ldots, L\}$, and the solution of (CP3) is given by $\breve{u}_{i}(t)=\breve{K}(t) \breve{x}_{i}(t)$. The result follows by combining the above two equations using (18).

Remark 1 Based on the definition of $\breve{x}_{i}(t)$, the control in (22) can be equivalently written as

$$
u_{i}(t)=-\breve{K}(t) x_{i}(t)-\sum_{\ell=1}^{L}\left(K^{\ell}(t)-\breve{K}(t)\right) x_{i}^{\ell}(t),
$$

where the first part represents a local state feedback and the second part represents offset terms proportional to eigen states.

Remark 2 Although the eigenstates $\left\{x_{i}^{\ell}(t)\right\}_{\ell=1}^{L}$ depend on the eigenvectors $\left(v^{1}, \ldots, v^{L}\right)$, the corresponding Riccati equations (20) only depend on the eigenvalues $\left(\lambda^{1}, \ldots, \lambda^{L}\right)$. So, if the coupling matrix has repeated eigenvalues, as is the case when there are certain symmetries in the graph $\mathcal{G}$, eigendirections with the same eigenvalue have the same Riccati equation. Therefore, we only need to solve $L_{\text {dist }}+1$, Riccati equations, where $L_{\text {dist }}$ denotes the number of distinct non-zero eigenvalues of the coupling matrix. 
Remark 3 The Riccati equtions (20)-(21) are significantly simpler to solve than the naive centralized Riccati equation. Each Riccati equation in (20)-(21) is of dimension $d_{x} \times$ $d_{x}$, while the centralized Riccati equation is of dimension $n d_{x} \times n d_{x}$. So, even if the coupling matrix is full rank (i.e., $L=n$ ) and all eigenvalues are distinct, solving the $n$ "one-dimensional" Riccati equations (20)-(21) is significantly simpler than solving one centralized " $n$-dimensional" Riccati equation. For graphs where $L \ll n$, these savings become even more drastic.

\section{B. Infinite horizon setup}

Let $Q^{\frac{1}{2}}$ denote the symmetric positive semi-definite matrix that satisfies $Q^{\frac{1}{2}}{ }^{\top} Q^{\frac{1}{2}}=Q$. For infinite horizon problems, we further impose the following standard assumptions.

(A3) $(A, B)$ is stabilizable and $\left(q_{0}^{\frac{1}{2}} Q^{\frac{1}{2}}, A\right)$ is detectable.

(A4) For all $\ell \in\{1, \ldots, L\},\left(A+\lambda^{\ell} D, B+\lambda^{\ell} E\right)$ is stabilizable and $\left(A+\lambda^{\ell} D, q^{\ell \frac{1}{2}} Q^{\frac{1}{2}}\right)$ is detectable .

Theorem 2 Suppose assumptions (A0)-(A4) hold. For $\ell \in$ $\{1, \ldots, L\}$, let $P^{\ell} \in \mathbb{R}^{d_{x} \times d_{x}}$ be the unique symmetric positive semi-definite solution to the algebraic Riccati equation

$$
\begin{aligned}
0= & \left(A+\lambda^{\ell} D\right)^{\top} P^{\ell}+P^{\ell}\left(A+\lambda^{\ell} D\right) \\
& -P^{\ell}\left(B+\lambda^{\ell} E\right)\left(r^{\ell} R\right)^{-1}\left(B+\lambda^{\ell} E\right)^{\top} P^{\ell}+q^{\ell} Q .
\end{aligned}
$$

Similarly, let $\breve{P} \in \mathbb{R}^{d_{x} \times d_{x}}$ be the unique symmetric positive semi-definite solution to the algebraic Riccati equation

$$
0=A^{\top} \breve{P}+\breve{P} A-\breve{P} B\left(r_{0} R\right)^{-1} B^{\top} \breve{P}+q_{0} Q .
$$

Then the optimal control strategy for Problem 2 is given by

$$
u_{i}(t)=-\breve{K} \breve{x}_{i}(t)-\sum_{\ell=1}^{L} K^{\ell} x_{i}^{\ell}(t)
$$

with $\breve{K}=\left(r_{0} R\right)^{-1} B^{\top} \breve{P}$ and $K^{\ell}=\left(r^{\ell} R\right)^{-1}\left(B+\lambda^{\ell} E\right)^{\top} P^{\ell}$.

The proof follows along the similar lines as the proof of Theorem 11 Under the extra assumptions (A3) and (A4), one only needs to replace the finite horizon costs with the infinite horizon costs and then solve decoupled LQR problems by solving the corresponding algebraic Riccati equations. (A3) and (A4) ensure the existence of solutions to the algebraic Riccati equations (24) and (23) (see e.g. [47]).

\section{Remarks on the information structure and the implementa- tion of the optimal strategy}

Since we are interested in regulating a deterministic system, we may implement the optimal control law either using openloop (i.e. pre-computed) control inputs or using closed-loop (i.e. state feedback) control inputs. For both implementations, the eigenvalues $\left\{\lambda^{\ell}\right\}_{\ell=1}^{L}$ need to be known at all subsystems.

For the open-loop implementation, one can write

$$
u_{i}(t)=-\breve{K}(t) \breve{\Phi}(t, 0) \breve{x}_{i}(0)-\sum_{\ell=1}^{L} K^{\ell}(t) \Phi^{\ell}(t, 0) x_{i}^{\ell}(0),
$$

where the state transition matrices $\breve{\Phi}(t, 0)$ and $\Phi^{\ell}(t, 0)$ are given by

$$
\begin{aligned}
\breve{\Phi}(t, 0) & =\exp \left(\int_{0}^{t}(A-B \breve{K}(\tau)) d \tau\right) \\
\Phi^{\ell}(t, 0) & =\exp \left(\int_{0}^{t}\left(A+\lambda^{\ell} D-\left(B+\lambda^{\ell} E\right) K^{\ell}(\tau)\right) d \tau\right) .
\end{aligned}
$$

Thus, to implement the control action, subsystem $i$ needs to know $\breve{x}_{i}(0)$ and $\left\{x_{i}^{\ell}(0)\right\}_{\ell=1}^{L}$, which can be obtained using one of the following three information structures:

1) All subsystems know the initial condition $x(0)$ and the eigendirections $\left\{v^{\ell}\right\}_{\ell=1}^{L}$. Using these, subsystem $i$ can compute $\left\{x_{i}^{\ell}(0)\right\}_{\ell=1}^{L}$ and $\breve{x}_{i}(0)$, and implement (26).

2) Subsystem $i, i \in \mathcal{N}$, knows its local initial state $x_{i}(0)$ and its local initial eigensystem states $\left\{x_{i}^{\ell}(0)\right\}_{\ell=1}^{L}$. Then subsystem $i$ can compute $\breve{x}_{i}(0)$ and implement (26).

3) All subsystems knows the initial state $\left\{x(0) v^{\ell}\right\}_{\ell=1}^{L}$. In addition, subsystem $i$ knows $v_{i}:=\left(v_{i}^{1}, \cdots, v_{i}^{L}\right)$ and its local initial state $x_{i}(0)$. Then subsystem $i$ can compute $\left\{x_{i}^{\ell}(0)\right\}_{\ell=1}^{L}$ and $\breve{x}_{i}(0)$, and implement (26).

The closed-loop implementation, which is given by (22) or (25), can be obtained by using one of the three information structures described above with $x(0), x_{i}(0)$ and $x_{i}^{\ell}(0)$ replaced by $x(t), x_{i}(t)$ and $x_{i}^{\ell}(t)$, respectively.

Furthermore, for the information structures in 2) and 3), a mixed implementation which combines open-loop and closeloop implementations can also be obtained via only replacing $x_{i}(0)$ by $x_{i}(t)$ in 2$)$ and 3$)$. In the mixed implementation, for any subsystem $i \in \mathcal{N}$, the close-loop part corresponds to the individual state $x_{i}(t)$ and the open-loop part corresponds to the terms $\left\{x_{i}^{\ell}(0)\right\}_{\ell=1}^{L}$ or $\left\{x(0) v^{\ell}\right\}_{\ell=1}^{L}$ which involve the aggregate of initial states of all subsystems.

\section{Applications to CONSEnsus}

Consensus refers to a distributed coordination problem in which nodes connected over a graph update their local states based on the states of their neighbors. The simplest objective is for all nodes to converge to a "consensus" value starting from any initial state $x(0)$, i.e.,

$$
\lim _{t \rightarrow \infty}\left\|x_{i}(t)-x_{j}(t)\right\|=0, \quad \forall i, j \in \mathcal{N} .
$$

There are various consensus protocols (i.e., rules to update the state at each node as a function of the state of the nearest neighbors and its own state), which have different rates of convergence. We refer readers to [7]-[9], [48] for an overview. Often these consensus protocols are hand crafted based on intuitions. In this section, we show that the standard consensus protocol naturally emerges as the optimal solution of an appropriately chosen networked control problem.

In particular, consider a (non-negatively) weighted connected undirected graph $\mathcal{G}(\mathcal{V}, \mathcal{E}, W)$ where $W$ represents its adjacency matrix. Now consider the system dynamics

$$
\dot{x}_{i}(t)=u_{i}(t), \quad i \in \mathcal{N}
$$

which is a special case of (1) with $A=0, B=I, D=0$, and $E=0$. Furthermore, consider the cost function

$$
c(x(t), u(t))=\langle x(t), Q x(t)\rangle_{M^{2}}+\langle u(t), R u(t)\rangle_{I}
$$


where $M=\operatorname{diag}\left(W \mathbb{1}_{n}\right)-W$ is the graph Laplacian matrix and $Q$ and $R$ are arbitrary symmetric positive definite matrices. It is well known that the rank of the Laplacian matrix of a (non-negatively weighted) connected graph is $n-1$ and all non-zero eigenvalues are positive. Thus, $L=n-1$ for this setup.

Lemma 1 The solution to Problem 2 with the dynamics in (29) and the cost in (30) is given by

$$
u_{i}(t)=-R^{-1} \Pi \sum_{\ell=1}^{n-1} \lambda^{\ell} x_{i}^{\ell}(t), \quad i \in \mathcal{N},
$$

where $\Pi$ denotes the symmetric positive semi-definite solution to $\Pi R^{-1} \Pi=Q$.

Proof Since $B=I, Q>0, R>0, q^{\ell}=\left(\lambda^{\ell}\right)^{2}>0, r^{\ell}=1$ $q_{0}=0$, and $r_{0}=1$, (A0)-(A4) are obviously satisfied. An application of Theorem 2 yields the following optimal control law

$$
u_{i}(t)=-\sum_{\ell=1}^{n-1} R^{-1} P^{\ell} x_{i}^{\ell}(t), \quad i \in \mathcal{N},
$$

where $P^{\ell}$ is the symmetric positive semi-definite solution to the algebraic Riccati equation

$$
0=-P^{\ell} R^{-1} P^{\ell}+\left(\lambda^{\ell}\right)^{2} Q .
$$

Note that $q_{0}=0$ in this example implies the solution to the auxiliary Riccati equation in (24) is $\breve{P}=0$. Hence $\breve{K}=0$ in (25) and the control law (32) does not contain the auxiliary part. Let $\Pi=\left(\lambda^{\ell}\right)^{-1} P^{\ell}$. Substituting $P^{\ell}$ in (33), $\Pi$ is then given by the symmetric positive semi-definite solution to $\Pi R^{-1} \Pi=Q$. Hence the optimal control law is given by (31).

Now, recall that

$$
\begin{aligned}
\sum_{\ell=1}^{n-1} \lambda^{\ell} x_{i}^{\ell}(t) & =x_{i}^{\mathcal{G}}(t)=\sum_{j \in \mathcal{N}} m_{j i} x_{j}=\sum_{j \in \mathcal{N}} m_{i j} x_{j} \\
& =\sum_{j \in \mathcal{N}} w_{i j}\left(x_{i}-x_{j}\right)
\end{aligned}
$$

Therefore, the optimal control may be written as

$$
u_{i}(t)=-R^{-1} \Pi \sum_{j \in \mathcal{N}} w_{i j}\left(x_{i}(t)-x_{j}(t)\right), \quad i \in \mathcal{N} .
$$

Thus the optimal control law is the same as the standard consensus protocol in [7]-[9]. A similar result was established in [49. Theorem 4.6], using a much more sophisticated proof argument.

\section{Generalizations to stochastic systems}

\section{A. Stochastic networked control problem}

In this section we consider a model similar to Section II-A but with stochastic dynamics. As before, there are $n$ subsystems that are connected over an undirected weighted graph $\mathcal{G}(\mathcal{N}, \mathcal{E}, W)$, with an associated symmetric coupling matrix $M$. For any $i \in \mathcal{N}$, the state $x_{i}(t)$, control $u_{i}(t)$, and the network fields $x_{i}^{\mathcal{G}}(t)$ and $u_{i}^{\mathcal{G}}(t)$ are defined as before. The difference is that rather than being deterministic, the system dynamics are stochastic and are given by

$d x_{i}(t)=\left[A x_{i}(t)+B u_{i}(t)+D x_{i}^{\mathcal{G}}(t)+E u_{i}^{\mathcal{G}}(t)\right] d t+F d w_{i}(t)$,

for all $i \in \mathcal{N}$, where the matrices $A, B, D, E$ and $F$ are as before, $F$ is a matrix of an appropriate dimension, the initial states $\left(x_{i}(0)\right)_{i \in \mathcal{N}}$ are deterministic, and $\left\{w_{i}(t) \in \mathbb{R}^{d_{w}}: i \in\right.$ $\mathcal{N}, t \geq 0\}$ are standard ( $d_{w}$-dimensional) Brownian motions that are independent across nodes.

As before, there is an instantaneous cost $c(x(t), u(t))$ for $t \in[0, T)$, and a terminal cost $c(x(T))$, given by (4) and (5).

Let $\mathcal{F}(t)$ denote the $\sigma$-algebra generated by $\{w(\tau): 0 \leq$ $\tau \leq t\}$ where $w(\tau) \triangleq \operatorname{cols}\left(w_{1}(\tau), \ldots, w_{n}(\tau)\right)$.

We are interested in the following optimization problem.

Problem 3 Choose an $\mathcal{F}(t)$-adapted control $u:[0, T) \rightarrow$ $\mathbb{R}^{d_{u} \times n}$ to minimize

$$
J(u)=\mathbb{E}\left[\int_{0}^{T} c(x(t), u(t)) d t+c_{T}(x(T))\right],
$$

subject to the system dynamics in (35) and initial conditions $\left(x_{i}(0)\right)_{i \in \mathcal{N}}$.

\section{B. Decompositions}

Recall that $w(t) \triangleq \operatorname{cols}\left(w_{1}(t), \ldots, w_{n}(t)\right) \in \mathbb{R}^{d_{w} \times n}$. We introduce the following noise processes in eigen directions and the auxiliary direction: for any $i \in \mathcal{N}$ and $\ell \in\{1, \ldots, L\}$,

$$
w_{i}^{\ell}(t) \triangleq w(t) v^{\ell} v_{i}^{\ell} \text { and } \breve{w}_{i}(t) \triangleq w_{i}(t)-\sum_{\ell=1}^{L} w_{i}^{\ell}(t) \text {. }
$$

The corresponding compact matrix representations are given by

$$
\begin{aligned}
\breve{w}(t) & \triangleq \operatorname{cols}\left(\breve{w}_{1}(t), \ldots, \breve{w}_{n}(t)\right), \\
w^{\ell}(t) & \triangleq \operatorname{cols}\left(w_{1}^{\ell}(t), \ldots, w_{n}^{\ell}(t)\right) .
\end{aligned}
$$

Clearly, $w^{\ell}(t)=w(t) v^{\ell} v^{\ell^{\top}}$ and $\mathbb{E}\left[w_{i}^{\ell}\right]=\mathbb{E}\left[\breve{w}_{i}\right]=0$.

Lemma 2 The following statements hold for all $t \in[0, T]$, $i, j \in\{1, \ldots, n\}, \ell, h \in\{1, \ldots, L\}$ :

1) $\breve{w}_{i}(t)$ and $w_{i}^{\ell}(t)$ are independent.

2) $\breve{w}_{j}(t)$ and $w_{i}^{\ell}(t)$ are independent if and only if $v_{j}^{\ell}\left(v_{j}^{\ell}-\right.$ $\left.v_{i}^{\ell}\right)=0$.

3) $\breve{w}_{i}(t)$ and $\breve{w}_{j}(t)$ are independent if and only if $v_{i}^{\ell} v_{j}^{\ell}=0$.

4) $w_{i}^{\ell}(t)$ and $w_{j}^{\ell}(t)$ are independent if and only if $v_{i}^{\ell} v_{j}^{\ell}=0$.

5) If $i \neq j$ and $\ell \neq h$, then $w_{i}^{\ell}(t)$ and $w_{j}^{h}(t)$ are independent.

PROOF Since for any fixed time $t \in[0, T], \breve{w}_{i}(t), \breve{w}_{j}(t), w_{j}^{\ell}(t)$ and $w_{k}^{h}(t)$ are Gaussian random variables with zero mean, they are independent if and only if the covariance matrix is zero. By explicitly computing the covariance matrices, results in Lemma 2] are verified. 
Since $w_{i}^{\ell}$ and $\breve{w}_{i}$ are linear combinations of independent standard Brownian motions, they themselves are Brownian motions. It is easy to verify that for $s>0, t \geq 0$,

$$
\begin{aligned}
& \operatorname{var}\left(w_{i}^{\ell}(t+s)-w_{i}^{\ell}(t)\right)=s\left(v_{i}^{\ell}\right)^{2} I_{d_{w}}, \\
& \operatorname{var}\left(\breve{w}_{i}(t+s)-\breve{w}_{i}(t)\right)=s\left(1-\sum_{\ell=1}^{L}\left(v_{i}^{\ell}\right)^{2}\right) I_{d_{w}} .
\end{aligned}
$$

Hence the intensities of $w_{i}^{\ell}$ and $\breve{w}_{i}$ are $\left|v_{i}^{\ell}\right|$ and $(1-$ $\left.\sum_{\ell=1}^{L}\left(v_{i}^{\ell}\right)^{2}\right)^{\frac{1}{2}}$, respectively.

Recall the definition of $\breve{x}_{i}(t), \breve{u}_{i}(t), x^{\ell}(t)$ and $u^{\ell}(t)$. Following arguments similar to the deterministic case, we obtain the following stochastic differential equations for the decomposed dynamics

$d x_{i}^{\ell}(t)=\left[\left(A+\lambda^{\ell} D\right) x_{i}^{\ell}(t)+\left(B+\lambda^{\ell} E\right) u_{i}^{\ell}(t)\right] d t+F d w_{i}^{\ell}(t)$

$d \breve{x}_{i}(t)=\left[A \breve{x}_{i}(t)+B \breve{u}_{i}(t)\right] d t+F d \breve{w}_{i}(t)$,

for all $i \in \mathcal{N}, \ell \in\{1, \ldots, L\}$. Following the proof argument of Proposition 2, we obtain

$$
J(u)=\sum_{i \in \mathcal{N}}\left[\breve{J}_{i}\left(\breve{u}_{i}\right)+\sum_{\ell=1}^{L} J_{i}^{\ell}\left(u_{i}^{\ell}\right)\right],
$$

where for all $i \in \mathcal{N}$ and $\ell \in\{1, \ldots, L\}$,

$$
\begin{gathered}
J_{i}^{\ell}\left(u_{i}^{\ell}\right)=\mathbb{E}\left[\int_{0}^{T}\left(q^{\ell} x_{i}^{\ell}(t)^{\top} Q x_{i}^{\ell}(t)+r^{\ell} u_{i}^{\ell}(t)^{\top} R u_{i}^{\ell}(t)\right) d t\right. \\
\left.\quad+q^{\ell} x_{i}^{\ell}(T)^{\top} Q_{T} x_{i}^{\ell}(T)\right], \\
\breve{J}_{i}\left(\breve{u}_{i}\right)=\mathbb{E}\left[\int_{0}^{T}\left(q_{0} \breve{x}_{i}(t)^{\top} Q \breve{x}_{i}(t)+r_{0} \breve{u}_{i}(t)^{\top} R \breve{u}_{i}(t)\right) d t\right. \\
\left.+q_{0} \breve{x}_{i}(T)^{\top} Q_{T} \breve{x}_{i}(T)\right] .
\end{gathered}
$$

\section{Optimal control solution}

Theorem 3 Under assumptions (A0), (A1) and (A2), the optimal control strategy for Problem 3 is the same as the strategy in Theorem 1 given by (22). Furthermore, the optimal cost is given by

$$
V(x(0))=\sum_{i \in \mathcal{N}}\left[\breve{V}_{i}\left(\breve{x}_{i}(0)\right)+\sum_{\ell=1}^{L} V_{i}^{\ell}\left(x_{i}^{\ell}(0)\right)\right],
$$

where for $i \in \mathcal{N}$ and $\ell \in\{1, \ldots, L\}$,

$$
\begin{aligned}
\breve{V}_{i}\left(\breve{x}_{i}(0)\right)=\breve{x}_{i}(0)^{\top} \breve{P}(0) \breve{x}_{i}(0) & \\
& +\left[1-\sum_{\ell=1}^{L}\left(v_{i}^{\ell}\right)^{2}\right] \int_{0}^{T} \operatorname{Tr}\left[\breve{P}(t) F F^{\top}\right] d t \\
V_{i}^{\ell}\left(x_{i}^{\ell}(0)\right)= & x_{i}^{\ell}(0)^{\top} P^{\ell}(0) x_{i}^{\ell}(0) \\
& +\left(v_{i}^{\ell}\right)^{2} \int_{0}^{T} \operatorname{Tr}\left[P^{\ell}(t) F F^{\top}\right] d t
\end{aligned}
$$

Proof The dynamics in 35 can be decomposed into 37 and (38), and the decomposition of the cost in (36) follows
(39), (40) and 441). Therefore, Problem 3 can be equivalently decomposed into the linear quadratic control problems defined by (37) and (40), and the linear quadratic control problems given by (38) and (41), where $i \in \mathcal{N}$. Note that the Brownian motions are not necessarily independent across all the decoupled problems as illustrated in Lemma 2 However, following the certainty equivalence principle for linear quadratic Gaussian problems (see e.g., [50]), we obtain the same optimal control feedback gain as the deterministic case, which does not depend on the intensity of the Brownian motion. This, together with the non-negativity of each term in (39) under assumptions (A1) and (A2), implies that solving the decomposed linear quadratic control problems independently yields the optimal feedback gain for and hence optimal solution to Problem 3 Therefore, the optimal feedback gains are the same as those in Theorem 11 for the linear quadratic control problems and the optimal control is given by (22). The optimal costs for the decomposed linear quadratic control problems are given by (43) and (44) (see for instance [51]) and hence the optimal cost for Problem 3 is given by (42).

Note that the intensity of the Brownian motion does not influence the optimal feedback gain but it effects the optimal cost under optimal control.

Remark 4 The result of Theorem 3 generalizes to the infinite horizon long run average cost setup and the infinite horizon discounted cost setup in a natural manner. For each of these setups, the optimal control law will be of the same form as Theorem 3 but the control gains will be time homogeneous and determined by the solution of an algebraic Riccati equation.

\section{ILLUSTRATIVE EXAMPLES}

\section{A. Adjacency matrix coupling}

Consider a network with $n=4$ subsystems connected over a graph $\mathcal{G}$, as shown in Fig. 2, with its adjacency matrix as the coupling matrix $M$. Note that $L=\operatorname{rank}(M)=2$. Consider

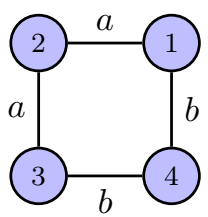

$$
\left[\begin{array}{llll}
0 & a & 0 & b \\
a & 0 & a & 0 \\
0 & a & 0 & b \\
b & 0 & b & 0
\end{array}\right]
$$

Fig. 2: Graph $\mathcal{G}$ with $n=4$ nodes and its adjacency matrix

the following couplings in the cost

$$
G=I-2 M+M^{2} \quad \text { and } \quad H=I .
$$

For the ease of notation define $\rho=\sqrt{\left(a^{2}+b^{2}\right) / 2}$ and $\theta=\tan ^{-1}(b / a)$. Then it is easy to verify that the nonzero eigenvalues of $M$ are $\lambda^{1}=-\rho$ and $\lambda^{2}=\rho$. The corresponding eigenvectors are $v^{1}=\left[-\frac{1}{2} \frac{\sin (\theta)}{\sqrt{2}}-\frac{1}{2} \frac{\cos (\theta)}{\sqrt{2}}\right]^{\top}$ and $v^{2}=\left[\frac{1}{2} \frac{\sin (\theta)}{\sqrt{2}} \frac{1}{2} \frac{\cos (\theta)}{\sqrt{2}}\right]^{\top}$. Observe that $q^{\ell}=\left(1-\lambda^{\ell}\right)^{2}$ is non-negative and $r^{\ell}=1$ is strictly positive, $\ell \in\{1,2\}$. Thus the model satisfies assumption (A2).

To illustrate how to use the result of Theorem 11 let's pick a subsystem, say subsystem 1 , and consider the calculations 
that need to be carried out at that subsystem. Recall that for all $i \in \mathcal{N}, x_{i}^{\ell}(0)=x(0) v^{\ell} v_{i}^{\ell}$. Thus

$$
\begin{aligned}
& x_{1}^{1}(0)=\frac{1}{4} x_{1}(0)-\frac{\sin (\theta)}{2 \sqrt{2}} x_{2}(0)+\frac{1}{4} x_{3}(0)-\frac{\cos (\theta)}{2 \sqrt{2}} x_{4}(0), \\
& x_{1}^{2}(0)=\frac{1}{4} x_{1}(0)+\frac{\sin (\theta)}{2 \sqrt{2}} x_{2}(0)+\frac{1}{4} x_{3}(0)+\frac{\cos (\theta)}{2 \sqrt{2}} x_{4}(0) .
\end{aligned}
$$

Following the mixed implementation with information structure 3) described in Section IV-A, subsystem 1 can calculate the trajectory for $x_{1}^{1}(t), x_{1}^{2}(t), t \in(0, T]$ based on the initial conditions. This together with real time local observation $x_{1}(t)$ yields $\breve{x}_{1}(t)$.

Subsystem 1 solves three Riccati equations to compute $P^{1}(t), P^{2}(t)$, and $\breve{P}(t)$ for $t \in[0, T]$, where

$$
\begin{aligned}
& -\dot{\breve{P}}(t)=A^{\top} \breve{P}(t)+\breve{P}(t) A-\breve{P}(t) B R^{-1} B^{\top} \breve{P}(t)+Q, \\
& -\dot{P}^{1}(t)=(A-\rho D)^{\top} P^{1}(t)+P^{1}(t)(A-\rho D) \\
& -\quad P^{1}(t)(B-\rho E) R^{-1}(B-\rho E)^{\top} P^{1}(t)+(1+\rho)^{2} Q \\
& -\dot{P}^{2}(t)=(A+\rho D)^{\top} P^{2}(t)+P^{2}(t)(A+\rho D) \\
& -\quad P^{2}(t)(B+\rho E) R^{-1}(B+\rho E)^{\top} P^{2}(t)+(1-\rho)^{2} Q
\end{aligned}
$$

with $\breve{P}(T)=Q_{T}, P^{1}(T)=(1+\rho)^{2} Q_{T}$ and $P^{2}(T)=(1-$ $\rho)^{2} Q_{T}$, and then applies the optimal control action given by

$$
\begin{aligned}
u_{1}(t)=-R^{-1}\left(B^{\boldsymbol{\top}} \breve{P}(t) \breve{x}_{1}(t)\right. & +(B-\rho E)^{\boldsymbol{\top}} P^{1}(t) x_{1}^{1}(t) \\
& \left.+(B+\rho E)^{\boldsymbol{\top}} P^{2}(t) x_{1}^{2}(t)\right)
\end{aligned}
$$

according to Theorem 11 Similar implementations hold for other subsystems.

Note that if each $x_{i}(t) \in \mathbb{R}^{d_{x}}$ then $x(t) \in \mathbb{R}^{4 d_{x}}$. A naive centralized optimal solution of the above system would involve solving a $4 d_{x} \times 4 d_{x}$-dimensional Riccati equation. In contrast, the above solution involves solving three $d_{x} \times d_{x}$-dimensional Riccati equations.

Moreover, these computational savings may increase with the size of the networks. For example, consider the graph $\mathcal{G}_{4 c}=\mathcal{G} \otimes \mathcal{K}_{c}$ with $4 c$ nodes, where $\mathcal{G}$ is the 4-node graph shown in Fig. 2 and $\mathcal{K}_{c}$ is the complete graph with $c$ nodes and each edge weight is $\frac{1}{c}$ where $c$ is a positive integer. The adjacency matrix of $\mathcal{G}_{4 c}$ is given by $M_{4 c}=M \otimes K_{c}$, where $M$ and $K_{c}=\frac{1}{c} \mathbb{1}_{c \times c}$ are the adjacency matrices of graph $\mathcal{G}$ and $\mathcal{K}_{c}$ respectively. The only non-zero eigenvalue of $K_{c}$ is 1 . Thus, the eigenvalues of $M_{4 c}$ are the same as eigenvalues of $M$. Note that the Riccati equations in Theorem 1 only depend on the eigenvalues. So for all different graphs $\mathcal{G}_{4 c}$ where $c$ can be any positive integer, the Riccati equations are the same, given by (46)! The method proposed in Theorem 11 would require solving the same three $d_{x} \times d_{x}$-dimensional Riccati equations in (46), while a naive direct solution would require solving a $4 c d_{x} \times 4 c d_{x}$-dimensional Riccati equation.

As an illustration, we consider the graph $\mathcal{G}_{4 c}=\mathcal{G} \otimes \mathcal{K}_{c}$ where $\mathcal{G}$ is given in Fig. 2 with weights $a=2$ and $b=1$. Recall that $G$ and $H$ are given by (45). As argued above, the matrix $M_{4 c}$ has two non-zero eigenvalues and the optimal control at each subsystem can be obtained by solving only
3 Riccati equations. Let us set $c=5$. Then $M_{20}=M \otimes \frac{1}{5} \mathbb{1}_{5 \times 5}$.

Example 1: Consider Problem 1 with 20 subsystems on $G_{20}$ where $d_{x}=d_{u}=1$, the coupling matrix is the adjacency matrix $M_{20}$ of the graph $\mathcal{G}_{20}$, and the parameters are $A=$ $2, B=1, D=3, E=0.5, Q=5, Q_{T}=6, R=2, T=2$. The evolutions of eigenstates and auxiliary states along with the corresponding eigencontrols and auxiliary controls are shown in Fig. 3 .

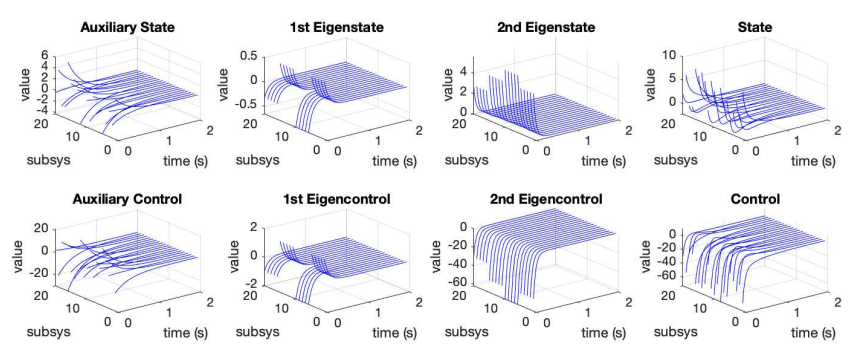

Fig. 3: Numerical example under the proposed optimal control on a network of size 20 over $[0, T]$ with $T=2$.

Example 2: To illustrate the case with higher dimensional local states, we consider a network of coupled harmonic oscillators where for subsystem $i \in \mathcal{N}$, the state is given by $x_{i}=\left[\theta_{i}, \omega_{i}\right]^{\top}$ representing the angle and angular velocity, and the control $u_{i}$ represents the input force. Thus $d_{x}=2$ and $d_{u}=1$. Consider Problem 1 with 20 coupled harmonic oscillators on $G_{20}$ where the coupling matrix is the adjacency matrix $M_{20}$ of the graph $G_{20}$ and the parameters are

$$
\begin{aligned}
& A=\left[\begin{array}{cc}
0 & 10 \\
-20 & 0
\end{array}\right], B=\left[\begin{array}{c}
0 \\
1.5
\end{array}\right], D=\left[\begin{array}{ll}
1 & 0 \\
0 & 1
\end{array}\right], R=1, \\
& E=\left[\begin{array}{l}
1 \\
1
\end{array}\right], Q=\left[\begin{array}{ll}
6 & 0 \\
0 & 6
\end{array}\right], Q_{T}=\left[\begin{array}{ll}
5 & 0 \\
0 & 5
\end{array}\right], T=2 .
\end{aligned}
$$

The result is illustrated in Fig. 4 ,

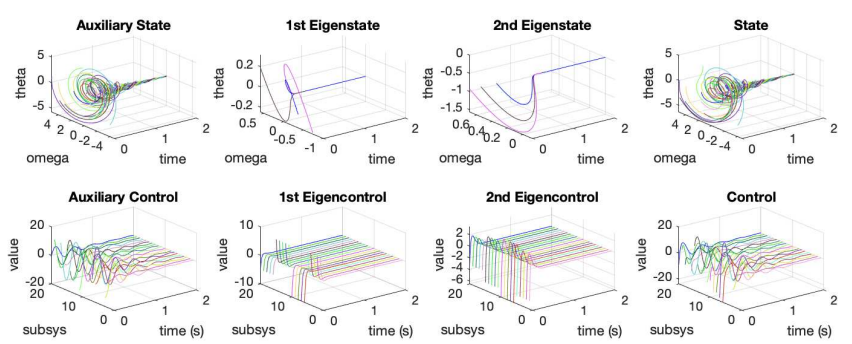

Fig. 4: Numerical example under the proposed optimal control on a network of 20 coupled harmonic oscillators with $T=2$.

\section{B. Adjacency matrix coupling for stochastic systems}

We consider the same model as in the previous section, but assume that the system dynamics are stochastic. In particular, we consider the graph $\mathcal{G}_{20}$ in Section VII-A

Example 3: Consider the stochastic generalization of Example 1 with $F=1$. All other parameters are the same 
as those in Example 1. The simulation result is given in Fig. 5.

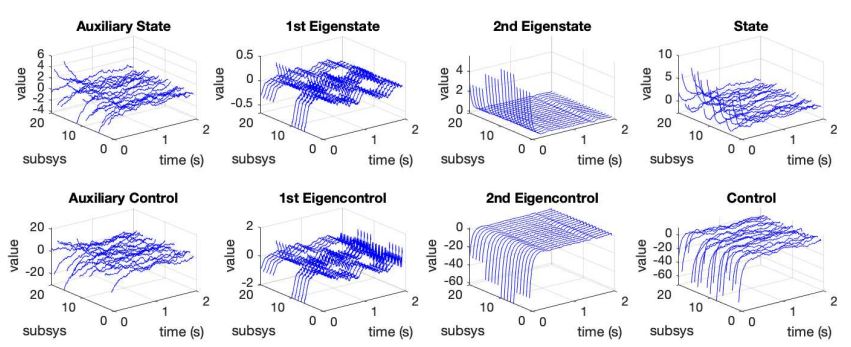

Fig. 5: Numerical example with additive noise under the proposed optimal control on a network of size 20 over $[0, T]$ with $T=2$.

Example 4: Consider the stochastic generalization of Example 2 with $F=\left[\begin{array}{ll}1 & 0 \\ 0 & 1\end{array}\right]$. All other parameters are the same as those in Example 2. The simulation result is given in Fig. 6.

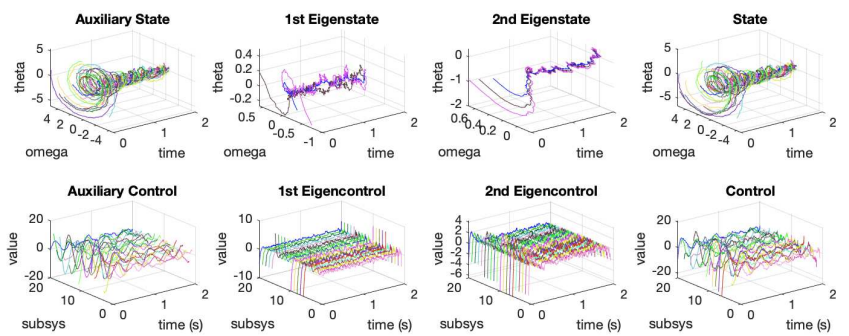

Fig. 6: Numerical example with additive noise under the proposed optimal control on a network of 20 coupled harmonics oscillators with time horizon $T=2$.

\section{Laplacian matrix coupling}

We now consider examples where the coupling matrix $M$ is the Laplacian matrix of the underlying graph $\mathcal{G}_{20}$ in Section VII-A

Example 5: Consider Problem 1 with 20 subsystems on $\mathcal{G}_{20}$ where $d_{x}=d_{u}=1$, the coupling matrix is the Laplacian matrix $\mathcal{L} \triangleq \operatorname{diag}\left(M_{20} \mathbb{1}_{20}\right)-M_{20}$ of the graph $\mathcal{G}_{20}$, and the parameters are $A=0.1, B=1, D=E=0, R=0.1$, $Q=1, Q_{T}=0, T=2, G=\mathcal{L}^{2}$ and $H=I$. The graph $\mathcal{G}_{20}$ is connected and hence the rank of $\mathcal{L}$ is 19 . However, there are only 5 distinct non-zero eigenvalues. Therefore, the solution following Theorem 1 requires solving $5+1$ decoupled scalar Riccati equations (see Remark 2): for $\ell \in\{1, \ldots, 5\}$

$$
-\dot{P}^{\ell}(t)=0.2 P^{\ell}(t)-10 P^{\ell}(t)^{2}+\left(\lambda_{\text {dist }}^{\ell}\right)^{2}, P^{\ell}(T)=0,
$$

where $\left\{\lambda_{\text {dist }}^{\ell}\right\}_{\ell=1}^{5}$ are the distinct non-zero eigenvalues of $\mathcal{L}$ and

$$
-\dot{\breve{P}}(t)=0.2 \breve{P}(t)-10 \breve{P}(t)^{2}+0, \quad \breve{P}(T)=0 .
$$

Note that, the solution to the auxiliary Riccati equation (48) is $\breve{P}(t)=0$ for all $t \in[0, T]$, which implies the control signal in the auxiliary direction should alway be zero (see the auxiliary control in Fig. 77. In contrast to the above, a direct centralized solution requires solving a $20 \times 20$ dimensional matrix Riccati equation. The simulation result is given in Fig. 7
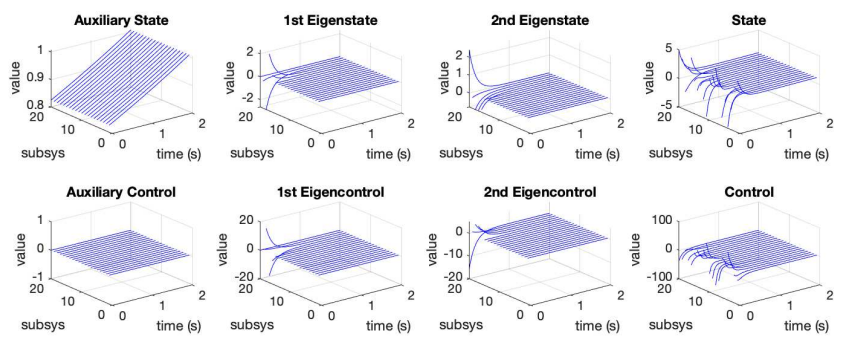

Fig. 7: Numerical example with Laplacian matrix coupling under the proposed optimal control over $[0, T]$ with $T=2$.

Example 6: Consider Problem 1 with 20 coupled harmonic oscillators on $\mathcal{G}_{20}$ where $d_{x}=2, d_{u}=1$, the coupling matrix is the Laplacian matrix $\mathcal{L} \triangleq \operatorname{diag}\left(M_{20} \mathbb{1}_{20}\right)-M_{20}$ of the graph $\mathcal{G}_{20}$, and the parameters are given by $G=\mathcal{L}^{2}, H=I$ and (47). The result is illustrated in Fig. 8.

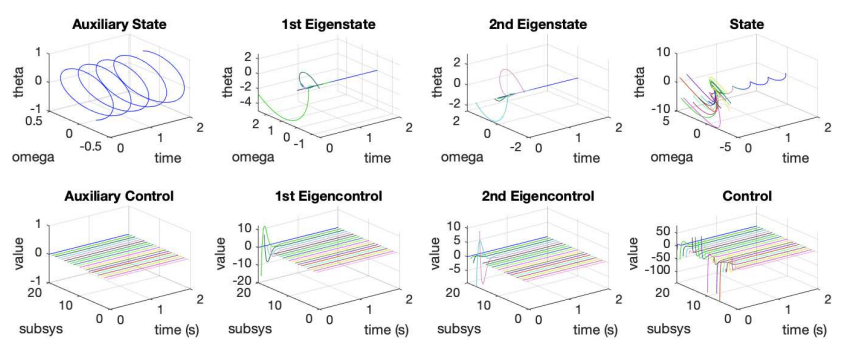

Fig. 8: Numerical example with Laplacian matrix coupling under the proposed optimal control on a network of 20 coupled harmonics oscillators over the time horizon $T=2$.

\section{A special case: mean-field coupling}

Suppose the graph $\mathcal{G}$ is a complete graph with all edge weights equal to $\frac{1}{n}$. Let $M$ be its adjacency matrix. Then $M=$ $\frac{1}{n} \mathbb{1}_{n \times n}$ has rank 1 and $\lambda_{1}=1$ is the only non-zero eigenvalue with the normalized eigenvector $v^{1}=\frac{1}{\sqrt{n}}[1, \ldots, 1]^{\top}$. Then $x^{1}(t)=x(t) v^{1} v^{1^{\top}}=x(t) M$. Thus, the eigenstate $x_{i}^{1}(t)=$ $\frac{1}{n} \sum_{j=1}^{n} x_{j}(t), i \in \mathcal{N}$, is the same for all subsystems and we denote it by $\bar{x}(t)$. Moreover, $q^{1}=\sum_{k=0}^{K_{G}} q_{k}:=\bar{q}$ and $r^{1}=$ $\sum_{k=0}^{K_{H}} r_{k}:=\bar{r}$. According to Theorem 11 the Riccati equation of eigensystem is given by

$$
\begin{aligned}
-\dot{\bar{P}}(t) & =(A+D)^{\top} \bar{P}(t)+\bar{P}(t)(A+D) \\
& -\bar{P}(t)(B+E)(\bar{r} R)^{-1}(B+E)^{\top} \bar{P}(t)+\bar{q} Q,
\end{aligned}
$$

where $\bar{P}(t):=P^{1}(t)$ and the final condition $\bar{P}(T)=\bar{q} Q_{T}$. The Riccati equation for the auxiliary system is given by

$$
-\dot{\breve{P}}(t)=A^{\top} \breve{P}(t)+\breve{P}(t) A-\breve{P}(t) B\left(r_{0} R\right)^{-1} B^{\top} \breve{P}(t)+q_{0} Q
$$

with the final condition $\breve{P}(T)=q_{0} Q_{T}$. The optimal control strategy is given by $u_{i}(t)=-\breve{K}(t)\left(x_{i}(t)-\bar{x}(t)\right)-\bar{K}(t) \bar{x}(t)$, 
where $\breve{K}(t)=\left(r_{0} R\right)^{-1} B^{\top} \breve{P}(t)$ and $\bar{K}(t)=(\bar{r} R)^{-1}(B+$ $E)^{\top} \bar{P}(t)$.

The above result is similar in spirit to [25, Theorem 1 and Theorem 4], which were derived for discrete time systems.

\section{CONCLUSION}

We consider the optimal control of network-coupled subsystems where the dynamics and the cost couplings depend on an underlying undirected weighted graph. The main idea of a low-dimensional decomposition is to project the state $x(t)$ into $L$ orthogonal eigendirections where $L$ denotes the rank of the coupling matrix. This projection generates $\left\{x^{\ell}(t)\right\}_{\ell=1}^{L}$ and an auxiliary state $\breve{x}(t)=x(t)-\sum_{\ell=1}^{L} x^{\ell}(t)$. A similar decomposition is obtained for the control inputs. These $L+1$ components are decoupled both in dynamics and cost. Therefore, the optimal control input for each component can be obtained by solving decoupled Riccati equations.

The proposed approach requires solving at most $L+1$ Riccati equations, each of dimension $d_{x} \times d_{x}$. If furthermore the coupling matrix has repeated non-zero eigenvalues, then the proposed approach only requires solving $L_{\text {dist }}+1$ decoupled Riccati equations where $L_{\text {dist }}$ (with $L_{\text {dist }} \leq L \leq n$ ) denotes the number of distinct non-zero eigenvalues of the coupling matrix. In contrast, a direct centralized solution requires solving an $n d_{x} \times n d_{x}$-dimensional Riccati equation. Thus, even when $L_{\text {dist }}=n$, the proposed approach leads to considerable computational savings. These savings improve significantly when $L_{\text {dist }} \ll n$, as is the case for adjacency matrices for many real-world networks.

Future directions of this work include: 1) similar problems where different subsystems may have different parameters in the dynamics, 2) error bounds for approximate solutions when the coupling matrix only admits an approximate low-rank representation, 3) problems with non-linear local dynamics.

\section{REFERENCES}

[1] Y. Ho and S. Mitter, Directions in Decentralized Control, Many-Person Optimization, and Large Scale Systems. Plenum, New York, 1976.

[2] N. Sandell, P. Varaiya, M. Athans, and M. Safonov, "Survey of decentralized control methods for large scale systems," IEEE Trans. Autom. Control, vol. 23, no. 2, pp. 108-128, 1978.

[3] Y.-Y. Liu, J.-J. Slotine, and A.-L. Barabási, "Controllability of complex networks," Nature, vol. 473, no. 7346, pp. 167-173, 2011.

[4] G.-H. Yang and S.-Y. Zhang, "Structural properties of large-scale systems possessing similar structures," Automatica, vol. 31, no. 7, pp. 1011-1017, 1995.

[5] G. Yan, G. Tsekenis, B. Barzel, J.-J. Slotine, Y.-Y. Liu, and A.-L. Barabási, "Spectrum of controlling and observing complex networks," Nature Physics, vol. 11, no. 9, pp. 779-786, 2015.

[6] F. Pasqualetti, S. Zampieri, and F. Bullo, "Controllability metrics, limitations and algorithms for complex networks," IEEE Trans. Control Netw. Syst., vol. 1, no. 1, pp. 40-52, 2014.

[7] R. Olfati-Saber and R. M. Murray, "Consensus protocols for networks of dynamic agents," in Proc. American Control Conf., vol. 2. IEEE, 2003, pp. 951-956.

[8] R. Olfati-Saber, J. A. Fax, and R. M. Murray, "Consensus and cooperation in networked multi-agent systems," Proc. IEEE, vol. 95, no. 1, pp. 215-233, 2007.

[9] A. Jadbabaie, J. Lin, and A. S. Morse, "Coordination of groups of mobile autonomous agents using nearest neighbor rules," IEEE Trans. Autom. Control, vol. 48, no. 6, pp. 988-1001, 2003.

[10] K. H. Movric and F. L. Lewis, "Cooperative optimal control for multi-agent systems on directed graph topologies," IEEE Trans. Autom. Control, vol. 59, no. 3, pp. 769-774, 2013.
[11] Z. Lin, M. Broucke, and B. Francis, "Local control strategies for groups of mobile autonomous agents," IEEE Trans. Autom. Control, vol. 49, no. 4, pp. 622-629, 2004.

[12] M. K. Sundareshan and R. M. Elbanna, "Qualitative analysis and decentralized controller synthesis for a class of large-scale systems with symmetrically interconnected subsystems," Automatica, vol. 27, no. 2 pp. 383-388, 1991.

[13] G.-H. Yang and S.-Y. Zhang, "Decentralized control of a class of largescale systems with symmetrically interconnected subsystems," IEEE Trans. Autom. Control, vol. 41, no. 5, pp. 710-713, 1996.

[14] D. D. Šiljak and A. Zečević, "Control of large-scale systems: Beyond decentralized feedback," Annual Reviews in Control, vol. 29, no. 2, pp. 169-179, 2005.

[15] T. Bian, Y. Jiang, and Z.-P. Jiang, "Decentralized adaptive optimal control of large-scale systems with application to power systems," IEEE Trans. Ind. Electron., vol. 62, no. 4, pp. 2439-2447, 2015.

[16] A. Arenas, A. Díaz-Guilera, J. Kurths, Y. Moreno, and C. Zhou, "Synchronization in complex networks," Physics Reports, vol. 469, no. 3, pp. 93-153, 2008

[17] H. Gang and Q. Zhilin, "Controlling spatiotemporal chaos in coupled map lattice systems," Physical Review Letters, vol. 72, no. 1, p. 68, 1994.

[18] R. Grigoriev, M. Cross, and H. Schuster, "Pinning control of spatiotemporal chaos," Physical Review Letters, vol. 79, no. 15, p. 2795, 1997.

[19] X. F. Wang and G. Chen, "Pinning control of scale-free dynamical networks," Physica A: Statistical Mechanics and Its Applications, vol. 310, no. 3-4, pp. 521-531, 2002.

[20] J.-S. Li, "Ensemble control of finite-dimensional time-varying linear systems," IEEE Trans. Autom. Control, vol. 56, no. 2, pp. 345-357, 2011.

[21] J. Lunze, "Dynamics of strongly coupled symmetric composite systems," International Journal of Control, vol. 44, no. 6, pp. 1617-1640, 1986.

[22] J. Grizzle and S. Marcus, "The structure of nonlinear control systems possessing symmetries," IEEE Trans. Autom. Control, vol. 30, no. 3, pp. 248-258, 1985

[23] D. Madjidian and L. Mirkin, "Distributed control with low-rank coordination," IEEE Trans. Control Netw. Syst., vol. 1, no. 1, pp. 53-63, 2014.

[24] J. Arabneydi and A. Mahajan, "Team-optimal solution of finite number of mean-field coupled LQG subsystems," in Proc. Conf. Decision and Control, Dec 2015, pp. 5308-5313.

[25] — , "Linear quadratic mean field teams: Optimal and approximately optimal decentralized solutions," arXiv preprint arXiv:1609.00056v2, 2017.

[26] R. Elliott, X. Li, and Y.-H. Ni, "Discrete time mean-field stochastic linear-quadratic optimal control problems," Automatica, vol. 49, no. 11, pp. 3222-3233, 2013.

[27] L. Arditti, G. Como, and F. Fagnani, "Graphical games and decomposition," arXiv preprint arXiv:2003.13123, 2020.

[28] P. Benner, "Solving large-scale control problems," IEEE Control Systems Magazine, vol. 24, no. 1, pp. 44-59, 2004.

[29] S.-C. Chang, T.-S. Chang, and P. B. Luh, "A hierarchical decomposition for large-scale optimal control problems with parallel processing structure," Automatica, vol. 25, no. 1, pp. 77-86, 1989.

[30] S. C. Hamilton and M. E. Broucke, "Patterned linear systems," Automatica, vol. 48, no. 2, pp. 263-272, 2012.

[31] M. Huang, P. E. Caines, and R. P. Malhamé, "Individual and mass behaviour in large population stochastic wireless power control problems: centralized and nash equilibrium solutions," in Proc. Conf. Decision and Control, 2003, pp. 98-103.

[32] M. Huang, R. P. Malhamé, and P. E. Caines, "Large population stochastic dynamic games: closed-loop McKean-Vlasov systems and the nash certainty equivalence principle," Communications in Information \& Systems, vol. 6, no. 3, pp. 221-252, 2006.

[33] J.-M. Lasry and P.-L. Lions, "Jeux à champ moyen. i-le cas stationnaire," Comptes Rendus Mathématique, vol. 343, no. 9, pp. 619-625, 2006.

[34] T. Li and J.-F. Zhang, "Asymptotically optimal decentralized control for large population stochastic multiagent systems," IEEE Trans. Autom. Control, vol. 53, no. 7, pp. 1643-1660, 2008.

[35] M. Aoki, "Control of large-scale dynamic systems by aggregation," IEEE Trans. Autom. Control, vol. 13, no. 3, pp. 246-253, 1968.

[36] J. Jiao, H. L. Trentelman, and M. K. Camlibel, "A suboptimality approach to distributed linear quadratic optimal control," IEEE Trans. Autom. Control, vol. 65, no. 3, pp. 1218-1225, 2020.

[37] F. Borrelli and T. Keviczky, "Distributed LQR design for identical dynamically decoupled systems," IEEE Transactions on Automatic Control, vol. 53, no. 8, pp. 1901-1912, 2008. 
[38] S. Gao and P. E. Caines, "Graphon control of large-scale networks of linear systems," IEEE Trans. Autom. Control, November 2019.

[39] — - "Optimal and approximate solutions to linear quadratic regulation of a class of graphon dynamical systems," in Proc. Conf. Decision and Control, Nice, France, December 2019, pp. 8359-8365.

[40] F. L. Lewis, H. Zhang, K. Hengster-Movric, and A. Das, Cooperative control of multi-agent systems: optimal and adaptive design approaches. Springer Science \& Business Media, 2013.

[41] M. Udell and A. Townsend, "Why are big data matrices approximately low rank?" SIAM Journal on Mathematics of Data Science, vol. 1, no. 1 , pp. 144-160, 2019.

[42] K. Zhou, H. Zha, and L. Song, "Learning social infectivity in sparse lowrank networks using multi-dimensional hawkes processes," in Artificial Intelligence and Statistics, 2013, pp. 641-649.

[43] S. Gao and P. E. Caines, "Spectral representations of graphons in very large network systems control," in Proc. Conf. Decision and Control, Nice, France, December 2019, pp. 5068-5075.

[44] B. D. MacArthur, R. J. Sánchez-García, and J. W. Anderson, "Symmetry in complex networks," Discrete Applied Mathematics, vol. 156, no. 18 , pp. 3525-3531, 2008.

[45] D. Liberzon, Calculus of variations and optimal control theory: a concise introduction. Princeton University Press, 2011.

[46] M. Huang, P. E. Caines, and R. P. Malhamé, "Social optima in mean field LQG control: centralized and decentralized strategies," IEEE Transactions on Automatic Control, vol. 57, no. 7, pp. 1736-1751, 2012.

[47] V. Kucera, "A contribution to matrix quadratic equations," IEEE Trans. Autom. Control, vol. 17, no. 3, pp. 344-347, 1972.

[48] M. Mesbahi and M. Egerstedt, Graph theoretic methods in multiagent networks. Princeton University Press, 2010.

[49] Y. Cao and W. Ren, "Optimal linear-consensus algorithms: An LQR perspective," IEEE Transactions on Systems, Man, and Cybernetics, Part B (Cybernetics), vol. 40, no. 3, pp. 819-830, 2009.

[50] H. Van de Water and J. Willems, "The certainty equivalence property in stochastic control theory," IEEE Trans. Autom. Control, vol. 26, no. 5, pp. 1080-1087, 1981.

[51] J. Yong and X. Y. Zhou, Stochastic controls: Hamiltonian systems and HJB equations. Springer Science \& Business Media, 1999, vol. 43.

\section{APPENDIX}

\section{A. Preliminary properties of the state decomposition}

Lemma 3 Let $k$ be a positive integer $k$ and $\ell, \ell^{\prime} \in\{1, \ldots, L\}$. Then, we have the following:

(P1) $x^{\ell}(t) M=\lambda^{\ell} x^{\ell}(t)$ and $u^{\ell}(t) M=\lambda^{\ell} u^{\ell}(t)$.

(P2) $x^{\ell}(t) M^{k}=\left(\lambda^{\ell}\right)^{k} x^{\ell}(t)$ and $u^{\ell}(t) M^{k}=\left(\lambda^{\ell}\right)^{k} u^{\ell}(t)$.

(P3) $x^{\ell}(t) G=q^{\ell} x^{\ell}(t)$ and $u^{\ell}(t) H=r^{\ell} u^{\ell}(t)$.

(P4) $\breve{x}(t) M=0$ and $\breve{u}(t) M=0$.

(P5) $\breve{x}(t) M^{k}=0$ and $\breve{u}(t) M^{k}=0$.

(P6) $\breve{x}(t) G=q_{0} \breve{x}(t)$ and $\breve{u}(t) H=r_{0} \breve{u}(t)$.

(P7) $x(t) G=q_{0} \breve{x}(t)+\sum_{\ell=1}^{L} q^{\ell} x^{\ell}(t)$ and $u(t) G=r_{0} \breve{u}(t)+$ $\sum_{\ell=1}^{L} r^{\ell} u^{\ell}(t)$.

(P8) $\sum_{i \in \mathcal{N}} x_{i}^{\ell}(t)^{\top} Q x_{i}^{\ell^{\prime}}(t)=\delta_{\ell \ell^{\prime}} \sum_{i \in \mathcal{N}} x_{i}^{\ell}(t)^{\top} Q x_{i}^{\ell^{\prime}}(t)$, where $\delta_{\ell \ell^{\prime}}$ is the Kronecker delta function.

(P9) $\sum_{i \in \mathcal{N}} x_{i}(t)^{\top} Q x_{i}^{\ell}(t)=\sum_{i \in \mathcal{N}} x_{i}^{\ell}(t)^{\top} Q x_{i}^{\ell}(t)$ and $\sum_{i \in \mathcal{N}} u_{i}(t)^{\top} R u_{i}^{\ell}(t)=\sum_{i \in \mathcal{N}} u_{i}^{\ell}(t)^{\top} R u_{i}^{\ell}(t)$

ProOF We show the result for $\breve{x}(t)$. The result for $\breve{u}(t)$ follows from a similar argument.

Since $v^{1}, \ldots, v^{L}$ are orthonormal, from (8) we have $v^{\ell} v^{\ell^{\top}} M=\lambda^{\ell} v^{\ell} v^{\ell^{\top}}$, which implies (P1). (P2) follows immediately from (P1) and (P3) follows from (P2).

(P4) follows immediately from the definition of $\breve{x}(t)$, (13) and (P1). (P5) follows immediately from (P4) and (P6) follows from (P5).
(P7) follows from (17), (P3) and (P6). To prove (P8), we observe that (9) implies that

$$
\begin{aligned}
\sum_{i \in \mathcal{N}} x_{i}^{\ell}(t)^{\top} Q x_{i}^{\ell^{\prime}}(t) & =\sum_{i \in \mathcal{N}} v_{i}^{\ell} v^{\ell^{\top}} x(t)^{\top} Q x(t) v^{\ell^{\prime}} v_{i}^{\ell^{\prime}} \\
= & \left(\sum_{i \in \mathcal{N}} v_{i}^{\ell} v_{i}^{\ell^{\prime}}\right) v^{\ell^{\top}} x(t)^{\top} Q x(t) v^{\ell^{\prime}} .
\end{aligned}
$$

Since $v^{1}, \ldots, v^{L}$ is orthonormal, we get $\sum_{i \in \mathcal{N}} v_{i}^{\ell} v_{i}^{\ell^{\prime}}=$ $v^{\ell^{\top}} v^{\ell^{\prime}}=\delta_{\ell \ell^{\prime}}$. Substituting this in (50) completes the proof of (P8). To prove (P9) observe that

$$
\begin{aligned}
\sum_{i \in \mathcal{N}} x_{i}(t)^{\top} Q x_{i}^{\ell}(t) & =\sum_{i \in \mathcal{N}} x_{i}(t)^{\top} Q x(t) v^{\ell} v_{i}^{\ell} \\
& =\sum_{i \in \mathcal{N}} v_{i}^{\ell} x_{i}(t)^{\top} Q x(t) v^{\ell} \\
& =v^{\ell^{\top}} x(t)^{\top} Q x(t) v^{\ell} .
\end{aligned}
$$

From (50), we get that the expression in (51) is equal to $\sum_{i \in \mathcal{N}} x_{i}^{\ell}(t)^{\top} Q x_{i}^{\ell}(t)$.

Lemma 4 Let $P, x$, and $y$ be defined in (19). Let $P_{i}$ denote the $i$-th column of $P$. Then, we can write

$$
\langle x, y\rangle_{P}=\sum_{i \in \mathcal{N}} x_{i}^{\top} y P_{i} \quad \text { or } \quad\langle x, y\rangle_{P}=\sum_{j \in \mathcal{N}} P_{j}^{\top} x^{\top} y_{j} .
$$

ProOF The result follows immediately from the definition of $\langle x, y\rangle_{P}$.

\section{B. Proof for Proposition 2}

We consider the terms depending on $x(t)$. The term depending on $u(t)$ may be simplified in a similar manner.

From (17) and linearity of $\langle\cdot, \cdot\rangle_{G}$ in both arguments, we get

$$
\begin{aligned}
\langle x(t), Q x(t)\rangle_{G}= & \left\langle\breve{x}(t)+\sum_{\ell=1}^{L} x^{\ell}(t), Q\left(\breve{x}(t)+\sum_{\ell=1}^{L} x^{\ell}(t)\right)\right\rangle_{G} \\
= & \langle\breve{x}(t), Q \breve{x}(t)\rangle_{G}+2\left\langle\sum_{\ell=1}^{L} x^{\ell}(t), Q \breve{x}(t)\right\rangle_{G} \\
& +\left\langle\sum_{\ell=1}^{L} x^{\ell}(t), Q\left(\sum_{\ell=1}^{L} x^{\ell}(t)\right)\right\rangle_{G} \cdot
\end{aligned}
$$

From Lemma 4 and (P6), the first term of (52) simplifies to

$$
\langle\breve{x}(t), Q \breve{x}(t)\rangle_{G}=q_{0} \sum_{i \in \mathcal{N}} \breve{x}_{i}(t)^{\top} Q \breve{x}_{i}(t),
$$

and the second term simplifies to

$$
\begin{aligned}
& \left\langle\sum_{\ell=1}^{L} x^{\ell}(t), Q \breve{x}(t)\right\rangle_{G}=q_{0} \sum_{i \in \mathcal{N}} \sum_{\ell=1}^{L} x_{i}^{\ell}(t)^{\top} Q \breve{x}_{i}(t) \\
& \quad=q_{0} \sum_{\ell=1}^{L} \sum_{i \in \mathcal{N}} x_{i}^{\ell}(t)^{\top} Q\left(x_{i}(t)-\sum_{\ell^{\prime}=1}^{L} x_{i}^{\ell^{\prime}}(t)\right) \\
& \quad \stackrel{(a)}{=} q_{0} \sum_{\ell=1}^{L} \sum_{i \in \mathcal{N}}\left(x_{i}^{\ell}(t)^{\top} Q x_{i}^{\ell}(t)-x_{i}^{\ell}(t)^{\top} Q x_{i}^{\ell}(t)\right) \\
& =0,
\end{aligned}
$$


where (a) follows from (P8) and (P9). From Lemma 4 and (P3), the third term of (52) simplifies to

$$
\begin{aligned}
\left\langle\sum_{\ell=1}^{L}\right. & \left.x^{\ell}(t), Q\left(\sum_{\ell=1}^{L} x^{\ell}(t)\right)\right\rangle_{G} \\
= & \sum_{i \in \mathcal{N}} \sum_{\ell=1}^{L} x_{i}^{\ell}(t)^{\top} Q\left(\sum_{\ell^{\prime}=1}^{L} q^{\ell^{\prime}} x_{i}^{\ell^{\prime}}(t)\right) \\
= & \sum_{\ell=1}^{L} \sum_{i \in \mathcal{N}} x_{i}^{\ell}(t)^{\top} Q\left(\sum_{\ell^{\prime}=1}^{L} q^{\ell^{\prime}} x_{i}^{\ell^{\prime}}(t)\right) \\
& \stackrel{(b)}{=} \sum_{\ell=1}^{L} \sum_{i \in \mathcal{N}} q^{\ell} x_{i}^{\ell}(t)^{\top} Q x_{i}^{\ell}(t),
\end{aligned}
$$

where $(b)$ follows from (P8). We get the result by substituting (53)-(55) in (52). 